\title{
Comparative Analysis of the Dynamical Spectra of a Polarization of an Active Medium and an Electromagnetic Field in the Superradiant Heterolasers
}

\section{V. Kocharovsky¹, A. S. Gavrilov¹, E. R. Kocharovskaya ${ }^{1}$, A. V. Mishin ${ }^{1}$, I. S. Ryabinin ${ }^{1}$, A. F. Seleznev ${ }^{1}$, and V. V. Kocharovsky ${ }^{1,2}$}

${ }^{1}$ Institute of Applied Physics, Russian Academy of Science, Nizhny Novgorod, 603950 Russian Federation

${ }^{2}$ Department of Physics and Astronomy, Texas A\&M University, College Station, Texas 77843, USA

\section{Abstract}

The complicated pulsed generation regimes of a CW-pumped superradiant semiconductor laser are analyzed via the dynamical spectra of the dipole optical

Corresponding Author: VI. V. Kocharovsky kochar@appl.sci-nnov.ru

Received: 22 July 2018 Accepted: 9 September 2018 Published: 8 October 2018

Publishing services provided by Knowledge E

(c) VI. V. Kocharovsky et al. This article is distributed under the terms of the Creative Commons Attribution License, which permits unrestricted use and redistribution provided that the original author and source are credited.

Selection and Peer-review under the responsibility of the Breakthrough Directions of Scientific Research at MEPhl Conference Committee.

\section{Introduction}

As shown in the recent review [1], the experimental evidences of a super-fluorescence (collective spontaneous emission under a pulsed pumping) give prospects for the creation of the superradiant heterolasers with continuous-wave (CW) incoherent pumping. As is well-known, in this class of lasers, the superradiance condition reads $\omega_{p 0}^{\prime \prime}>$ $T_{2}^{-1}$, where $\omega_{p 0}^{\prime \prime} \approx \bar{n} v_{c}^{2} T_{E}-1 / T_{2}-1 / T_{2}^{*}$ is the growth rate of the most unstable hot mode, $v_{c}=\sqrt{2 \pi d^{2} \omega_{21} N_{0} \Gamma /\left(\varepsilon_{0} \hbar\right)}$ the cooperative frequency, $\bar{n}$ the population inversion (normalized to unity) of the active centers averaged over a cavity, $N_{0}$ their density, $T_{E}$ a photon lifetime for a given cavity mode, $\Gamma$ the mode-filling factor, $\varepsilon_{0}$ the dielectric (real) permittivity of a background medium (matrix), $\omega_{21}$ the central frequency of a spectral line of active centers, $d$ their dipole transition moment, $2 / T_{2}$ and $2 / T_{2}^{*}$ the homogeneous and inhomogeneous line broadening (see also [2,3]). The superradiant lasing is expected to be non-stationary and defined mainly by the dynamics of a oscillations of active centers. This novel approach appears to be more informative than the standard analysis of the dynamical spectra of laser emission if a dipole relaxation rate is less than a cavity relaxation rate. The advantages of the method are demonstrated for a number of superradiant lasing regimes on the basis of the numerical solution to $1 \mathrm{D}$ Maxwell-Bloch equations for a two-level active medium in a low-Q cavity within one-dimensional approximation. 
polarization of an active medium, because a necessary condition for the superradiance to occur is that a lifetime of a photon in a cavity is shorter than that of the polarization of active centers. Hence, it is suggestive to use a dynamical spectrum of polarization for the analysis of the generation regimes of a superradiant laser. We will show, for the first time to our knowledge, that this approach makes it possible a deeper physical insight into the nonlinear mode coupling and interaction of various groups of active centers. In this way, we can essentially enrich the analysis of the superradiant lasing regimes that was based previously on the studies of the dynamical spectra of the population inversion and output emission as well as on the use of the empirical modes with a variable spatial-temporal or spectral-temporal structure [4-7].

Let us illustrate the advantages of the proposed approach on the basis of the well-known semiclassical two-level model of an active medium and one-dimensional model of wave propagation in a cavity (see $[2,7,8]$ and references therein). Namely, let us use the semiclassical Maxwell-Bloch equations for the complex amplitudes, $a_{ \pm}=A_{ \pm} /\left(2 \pi d N_{0}\right)$, of counterpropagating waves of the electric field

$$
E(z, t)=\operatorname{Re}\left\{\left[A_{+}(z, t) \exp \left(i k_{0} z\right)+A_{-}(z, t) \exp \left(-i k_{0} z\right)\right] \exp \left(-i \omega_{0} t\right)\right\} \sqrt{\Gamma / \varepsilon_{0}},
$$

the complex amplitudes, $p_{ \pm}(\Delta)=P_{ \pm}(z, t, \Delta) /\left(d N_{0}\right)$, of the polarization spectral density of active centers (it is related via the normalization to a current dipole moment of an individual active center)

$$
P(\Delta, z, t)=\operatorname{Re}\left\{\left[P_{+}(z, t, \Delta) \exp \left(i k_{0} z\right)+P_{-}(z, t, \Delta) \exp \left(-i k_{0} z\right)\right] \exp \left(-i \omega_{0} t\right)\right\} f_{L}(\Delta) \sqrt{\varepsilon_{0} / \Gamma}
$$

and the smooth, $n$, and grating, $n_{z}$, components of the population inversion spectral density

$$
N(\Delta, z, t)=N_{0}\left\{n(z, t, \Delta)+\operatorname{Im}\left[n_{z}(z, t, \Delta) \exp \left(2 i k_{0} z\right)\right]\right\} f_{L}(\Delta) .
$$

We take into account the inhomogeneous broadening of the spectral line and describe its normalized profile with a Lorentzian $f_{L}(\Delta)=\Delta_{0} / \pi\left(\Delta^{2}+\Delta_{0}^{2}\right)$, where $\Delta=\left(\omega-\omega_{21}\right) / v_{c}$ is the normalized detuning of the transition frequency $\omega$ of an active center from the central line frequency $\omega_{21}$, and $\Delta_{0}=1 /\left(T_{2}^{*} v_{c}\right)$ the normalized inhomogeneous line half-width. We consider the simplest case when the spectral line center $\omega_{21}$ coincides with the distributed feedback (Bragg) resonance frequency, $\omega_{0}=k_{0} c / \varepsilon_{0}^{1 / 2}$. For simplicity, we assume that the matrix, sample, and occupation of the transverse mode with active centers are homogeneous $\left(\varepsilon_{0}, N_{0}, \Gamma=\right.$ const $)$ along the laser cavity of length $B,-B / 2<z<B / 2$, and neglect the ohmic, diffraction and waveguide radiation losses. We take into account the selection of longitudinal modes, which is essential, for 
example, for attaining superradiance in ensembles of active centers with inhomogeneously broadened spectral lines, assuming the presence of the distributed feedback (DFB) of counterpropagating waves due to the weak (with the amplitude $\beta<<1$ ) harmonic modulation (with spatial period $\pi / k_{0}=\lambda_{0} / 2$ ) of the matrix permittivity, $\varepsilon_{M}=$ $\varepsilon_{0} \operatorname{Re}\left[1+4 \beta \exp \left(2 i k_{0} z\right)\right]$. The modulation of the waveguide walls provides the same effect.

The one-dimensional integro-differential semiclassical equations of the spatialtemporal dynamics of the superradiant laser may be written out in the form $[1,5]$

$$
\begin{aligned}
& {[\partial / \partial \tau \pm \partial / \partial \zeta] a_{ \pm}=i \beta^{(*)} a_{\mp}+i \int p_{ \pm}(\Delta) f_{L}(\Delta) I^{-1 / 2} d \Delta,} \\
& {\left[\partial / \partial \tau+\Gamma_{2}+i \Delta\right] p_{ \pm}(\Delta)=-\sqrt{I}\left(i n(\Delta) a_{ \pm} \pm n_{z}^{(*)}(\Delta) a_{\mp} / 2\right),} \\
& {\left[\partial / \partial \tau+\Gamma_{1}\right]\left(n(\Delta)-n_{p}\right)=-\sqrt{I} \operatorname{Im}\left(a_{+} p_{+}^{*}(\Delta)+a_{-} p_{-}^{*}(\Delta)\right),} \\
& {\left[\partial / \partial \tau+\Gamma_{1}\right] n_{z}=\sqrt{I}\left(a_{-}^{*} p_{+}(\Delta)-a_{+} p_{-}^{*}(\Delta)\right),}
\end{aligned}
$$

where * denotes complex conjugation and a superscript $\left(^{*}\right)$ means no superscript for the upper sign and complex conjugation for the lower sign in the terms with $a_{\mp}$ factors. Here, the following dimensionless quantities are introduced: $I=v_{c}^{2} / \omega_{21}^{2}<<1 ; \tau=t v_{c}$ and $\zeta=z / B_{c}$ are the time (normalized to the cooperative frequency) and the longitudinal coordinate (normalized to the cooperative length, $B_{c}=c /\left(v_{c} \varepsilon_{0}^{1 / 2}\right)=1 /\left(k_{0} I^{1 / 2}\right)$ ); $\Gamma_{1,2}=1 /\left(v_{c} T_{1,2}\right)$ the normalized rates of inversion and polarization relaxation; $L=B / B_{c}$ is the normalized length of the sample, and $n_{p}$ the inversion of an individual two-level active center with the transition frequency $\omega=\omega_{21}+v_{c} \Delta$ produced by CW pumping ( $n_{p}=-1$ in the absence of pumping, max $\left|n_{p}\right|=1$ ).

\section{Active Medium with a Homogeneous Broadening of Spectral Line in a Fabry-Perot Cavity}

Let us consider the case of an active medium with a weak inhomogeneous broadening of spectral line, $\Delta_{0}<<\Gamma_{2}$, placed in a symmetric low-Q Fabry-Perot (FP) cavity with the equal reflection factors of the end mirrors, $R_{1}=R_{2}=R$. We choose some particular laser parameters, listed in Figure 1 caption, which allow us to compare an initial pulse of strong superfluorescence, the transient pulses of moderate superradiance, and a subsequent weak quasi-stationary lasing under CW pumping, $n_{p}=1$.

The first superfluorescence pulse (very similar for both output ends of the laser) is associated with a resonant symmetric electromagnetic mode, takes place due to an 
initial inversion, $n(t=0)=n_{p}$, appears with an essential delay, $t_{d} \approx 23$, has a very short duration, $\delta \mathrm{T}<<\mathrm{T}_{E} \approx 2<<\mathrm{T}_{2}$, does not depend on a weak pumping rate, $\Gamma_{1}=T_{1}^{-1}$, and shows a wide symmetric smooth spectrum occupying detunings $|\Delta|<1$ for both the polarization and the field at a laser facet. During the pulse, all active centers behave similar to each other, cooperatively, independently on their partial frequencies. After the pulse, a population of the upper energy level is almost completely exhausted (the inversion of active centers becomes close to -1 everywhere except the cavity center), and a self-consistent nonlinear transient dynamics of the center's ensemble and the field modes in a cavity starts only after a delay time of the order of the inverse pumping rate [7], $T_{1}=100$.
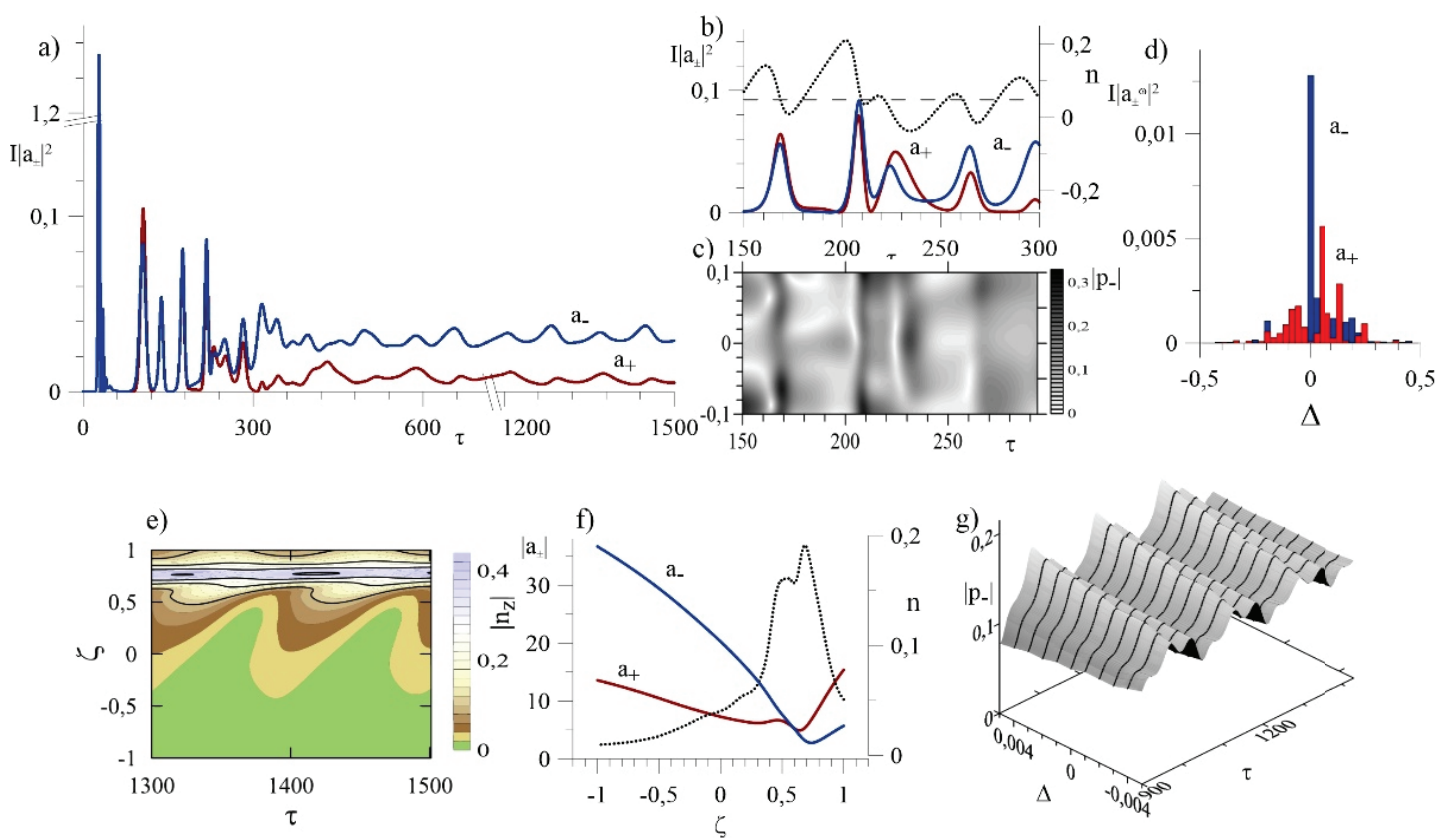

Figure 1: Weakly modulated superradiant lasing of a sample of length $L=2$ in a Fabry-Perot cavity with moderate reflection factors $R=0.37\left(T_{E}=2\right)$ in the case of CW pumping $\left(n_{p}=1\right)$ and an almost homogeneously broadened spectral line: $\Gamma_{2}=2 \Gamma_{1}=0.02, \Delta_{0}=0.002$. (a) Oscillograms of the normalized output radiation intensities, $I\left|a_{ \pm}\right|^{2}$. (b) The same enlarged oscillograms and an oscillogram of the average population inversion, $n$, (dotted curve) for the transient period of time $150<\tau<300$. (c) Dynamical spectrum of the polarization, $\left|p_{-}(\Delta, \tau)\right|$, at the left facet of an active sample during the transient superradiant phase. (d) Spectrum of the output fields at the left and right ends of laser. (e) Spatial-temporal evolution of the inversion grating, $\left|n_{z}(\zeta, \tau, \Delta=0)\right|$. (f) Spatial structure of the inversion, $n$, (dotted curve) and the fields propagating to the left $\left(a_{-}\right)$and right $\left(a_{+}\right)$. (g) Dynamical spectrum of the polarization, $\left|p_{-}(\Delta, \tau)\right|$, at $\zeta=-1$ during the developed lasing phase.

According to Figure 1a, there appear six superradiance pulses with a duration of each, $\delta \tau \sim 10-30$, less than the polarization relaxation time, $T_{2}=50$; see also Figure $1 \mathrm{~b}$, which shows four such pulses. Their amplitudes are several times less than the amplitude of the first superfluorescence pulse, and their spectra gradually become slightly asymmetric. These asymmetric pulse spectra are naturally imprinted and clearly seen in the dynamical spectrum of polarization (Figure $1 c$ ), though extraordinary numerical 
efforts are required to resolve this asymmetry from the spectral analysis of the field. In accord with the Kotelnikov-Nyquist-Shannon theorem, even a total spectrum of the field, taken for the whole transient period 75-300 and shown in Figure 1d, is rather rude and does not include the details of the asymmetry.

The dynamical spectral asymmetry is formed due to a quite strong inversion grating owing to the beating of the counterpropagating waves. At the time $t>300$ this grating becomes quasi-stationary and occupies mainly one half of a cavity in the vicinity of one end (left or right, see Figure 1e), so that there happens a spontoneous symmetry breaking of the field (Figure 1f) and polarization, and the amplitudes of output field at the opposite ends of the laser become different (see Figure 1a). The amplitude profiles of the counterpropagating waves in a cavity preserve their forms and oscillate slightly as a whole with a period $\approx 160$. The optical dipoles of active centers follow this quasi-adiabatic dynamics almost independently on their frequencies within a narrow inhomogeneously broadened line (Figure 19).

\section{Active Medium with a Homogeneous Broadening of Spectral Line in a DFB Cavity}

All described three stages - initial superfluorecsence, transient superradiance, and developed lasing - change completely if the same active medium under the same pumping is placed in a DFB cavity of the same quality (the same photon lifetime, $\left.T_{E}=2\right)$ for the main high-Q modes. In this case, the spontaneous symmetry breaking occurs from the very beginning because, contrary to the resonant one mode superfluorescence in a FP cavity, there are two equally important hot modes (with opposite frequency detunings $\Delta \approx \pm 0.2$ ) and their superfluorescent and subsequent superradiant behavior is strongly affected by initial (random) difference between their phases $[5]$.

The superfluorescence pulses at the left and right laser facets become essentially different (Figure 2a, c, d), depend on an initial noise (small amplitudes of field and polarization), and have two times longer typical durations and delay times. Their spectra have two times smaller widths and a pronounced dip at $|\Delta|<0.2$ that originates from the distributed Bragg reflections of counterpropagating waves with small frequency detuning. The polarization and population inversion of active centers again are insensitive to their frequencies within a narrow inhomogeneously broadened line and accorded well to the field due to the Bloch-vector conservation law that is valid for the time periods less than $T_{2}=50<T_{1}$. According to Figure $2 \mathrm{~b}$, the polarization 

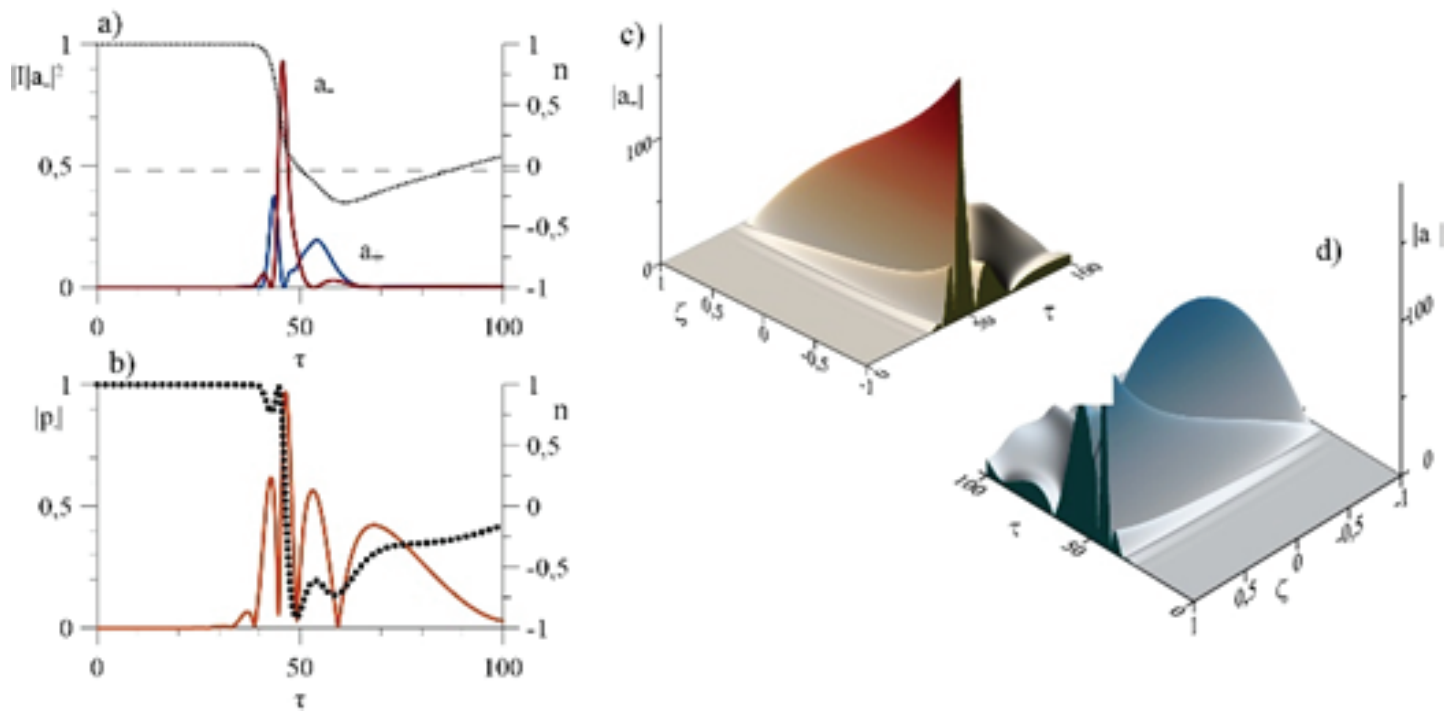

Figure 2: Oscillatory superfluorescence of a sample of length $L=2$ in a Bragg cavity with the DFB coefficient $\beta=1\left(T_{E}=2\right)$ in the case of CW pumping $\left(n_{p}=1\right)$ and an almost homogeneously broadened spectral line: $\Gamma_{2}=2 \Gamma_{1}=0.02, \Delta_{0}=0.002$. (a) Oscillograms of the normalized output radiation intensities, $I\left|a_{ \pm}\right|^{2}$, (the upper and lower solid curves correspond to $a_{-}$and $a_{+}$, respectively) and the averaged inversion $n$ (dotted curve). (b) Oscillograms of the polarization, $\left|p_{-}(\tau)\right|$, and the inversion, $|n(\tau)|$, at $\zeta=-1$ (dotted curve). (c, d) Spatial-temporal evolution of the amplitudes $\left|a_{-}(\zeta, \tau)\right|$ and $\left|a_{+}(\zeta, \tau)\right|$ of a wave running to the left and right, respectively.

at each laser facet is governed by the fields of both superradiant modes, though its response has an inertial character due to the finite lifetime $T_{2}$.

Note that the population inversion is modulated more sharply and deeper at the facets than at the center part of the laser, and the pumping makes growing the inversion monotonously everywhere in the cavity on a timescale $T_{1}=100$. During a transient period of time 200-1200, an average inversion becomes positive and high enough (though not close to +1 ) so that several moderate pulses of mode superradiance with a duration about 20-50, that is, less than $T_{2}$, are emitted irregularly (see Figure 3a). Due to the mode competition, this transient superradiant phase takes longer time than that in the case of FP cavity (cf. Figure 1a) and transforms smoothly to the developed lasing phase of quasi-periodic emission of mode superradiant pulses.

The intervals between short pulses of superradiant lasing vary from 50 to 150 , demonstrating an average period a bit greater than 100, and both the pulse durations and amplitudes vary in a couple of times from pulse to pulse (Figure za). The nonlinear interaction and nonadiabatic behavior (linear coupling) of modes in the presence of inhomogeneous and nonstationary population inversion of an active medium are responsible for the unstable pulsed lasing. Under these conditions, the phases of counterpropagating waves are highly variable and irregular in time and space, so 


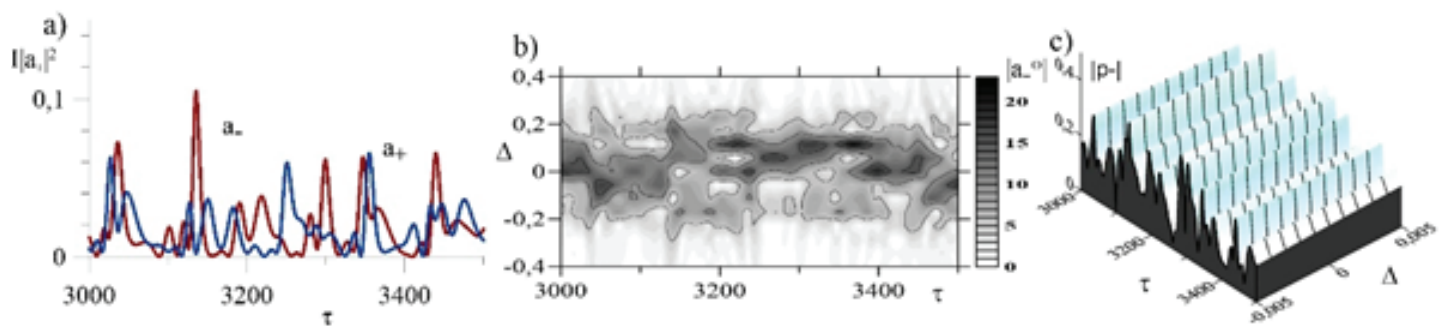

Figure 3: Superradiant lasing of a sample of length $L=2$ in a Bragg cavity with the DFB coefficient $\beta=1\left(T_{E}=2\right)$ in the case of CW pumping, $n_{p}=1$, and an almost homogeneously broadened spectral line: $\Gamma_{2}=2 \Gamma_{1}=0.02, \Delta_{0}=0.002$. (a) Oscillograms of the normalized output radiation intensities, $I\left|a_{ \pm}\right|^{2}$. Dynamical spectrum (b) of the field, $\left|a_{-}^{\omega}(\Delta, \tau)\right|$, and (c) of the polarization, $\left|p_{-}(\Delta, \tau)\right|$, at the left facet of an active sample.

that an inversion grating of an active medium is irregular, weak on average and does not influence much the coupling of waves and the lasing dynamics. As a result, the spontaneous symmetry breaking is clearly present during each superradiant pulse, but strongly suppressed on averaging over many pulses. The frequency asymmetry (with respect to $\Delta=0$ ) of the dynamical spectrum of field may exist during emission of several pulses (Figure $3 \mathrm{~b}$ ), but disappears for the long-term periods of time. However, the dynamical spectrum of polarization (and the corresponding field) in a narrow spectral line of an active medium, $\Delta<2 \Delta_{0}=0.01$, is almost symmetric (see Figure 3c). The spectral asymmetry of lasing may be important in the case of a strong inhomogeneous broadening of spectral line which is considered in the rest part of the article.
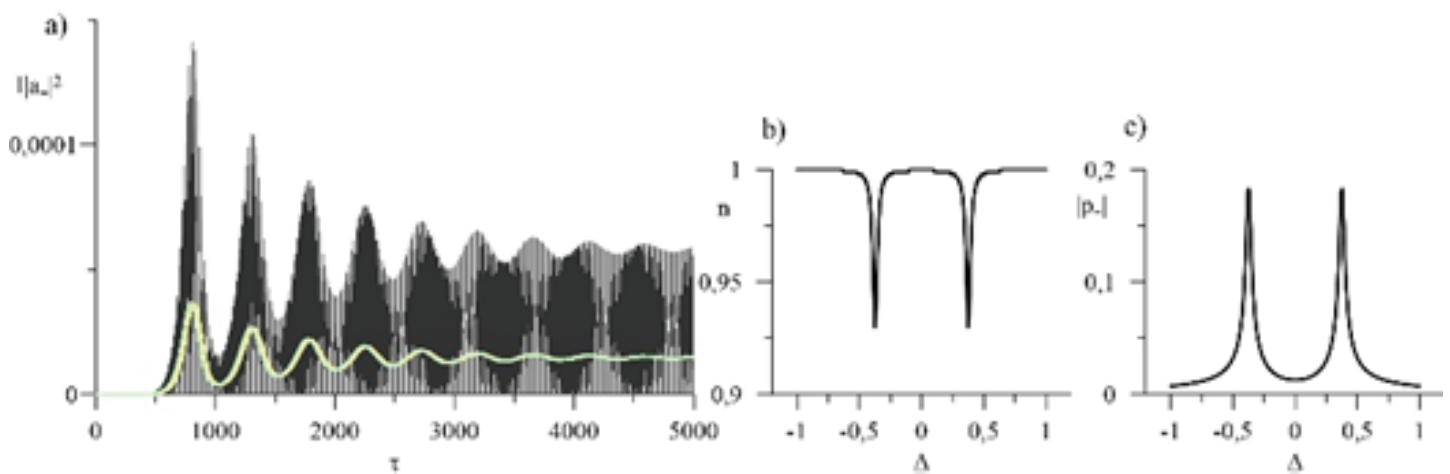

Figure 4: Lasing of a sample of length $L=5$ in a combined Fabry-Perot cavity with the complex reflection factor of both mirrors, $R=0.1 e^{i \pi / 2}$, and with the DFB coefficient $\beta=0.1\left(T_{E}=2.9\right)$ in the case of CW pumping $\left(n_{p}=1\right)$ and an inhomogeneously broadened spectral line: $\Gamma_{2}=2 \Gamma_{1}=0.02, \Delta_{0}=4$. (a) Oscillograms of the normalized output radiation intensities, $I\left|a_{ \pm}\right|^{2}$. Oscillograms of the fields of each of the two coherent modes are shown by light curves. (The dark background is caused by frequent intensity oscillations with a frequency of the order of the cooperative frequency.) (b) Spectrum of the inversion, $|n(\Delta, \tau=1500, \zeta=-L / 2)|$. (c) Spectrum of the polarization, $\left|p_{-}(\Delta, \tau=1500, \zeta=-L / 2)\right|$. 


\section{Active Medium with an Inhomogeneous Broadening of Spectral Line in a Combined DFB-FP Cavity with Symmetric Modes}

In the case of superradiant lasers with a strong inhomogeneous broadening of spectral line exceeding the cooperative frequency, when $\Delta_{0}>>1>T_{E}^{-1}>>T_{2}^{-1}$, there are at least five qualitatively different operation regimes of lasing: quasi-stationary, selfmodulation, regular pulsed, irregular pulsed with quasiperiodic pulse trains, and quasistochastic $[1,4,5]$. Which regime occurs and how it looks like depend on a cavity length $L$, the pumping level $n_{p}$ and rate $T_{1}$, and a spectrum of lasing modes.
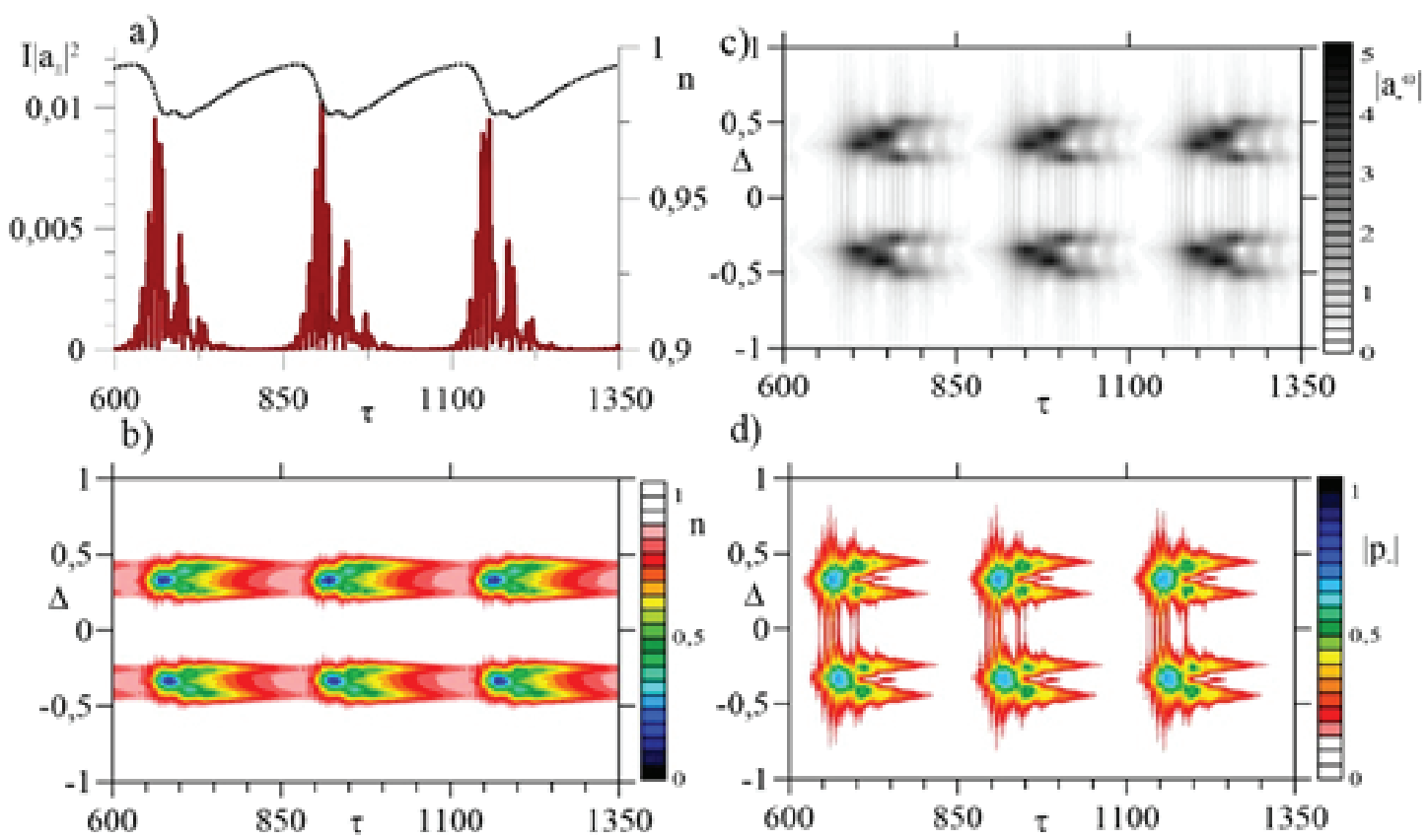

Figure 5: Superradiant lasing of a sample of length $L=6$ in a combined Fabry-Perot cavity with the complex reflection factor of both mirrors, $R=0.1 e^{i \pi / 2}$, and with the DFB coefficient $\beta=0.1\left(T_{E}=3.7\right)$ in the case of CW pumping $\left(n_{p}=1\right)$ and an inhomogeneously broadened spectral line: $\Gamma_{2}=2 \Gamma_{1}=0.02$, $\Delta_{0}=4$. (a) Oscillograms of the normalized output radiation intensities, $I\left|a_{ \pm}\right|^{2}$, and an oscillogram of the average inversion, $n$, (dotted curve). Dynamical spectrum (b) of the inversion, $|n(\Delta, \tau)|$, as well as (c) of the field, $\left|a_{-}^{\omega}(\Delta, \tau)\right|$, and (d) of the polarization, $\left|p_{-}(\Delta, \tau)\right|$, at the left facet of an active sample.

Figures 4 and 5 demonstrate the lasing features in the cases of quite short cavity lengths, $L=5$ and $L=6$, (which exceed a bit the lasing threshold value, $L_{t h} \sim 1 / \Delta_{0}$ ) and two symmetric hot modes situated at the deturnings $\Delta \approx \pm 0.4$ and fixed by choosing specific values of the amplitudes and phases of the mirror reflection factors in a combined DFB-FP cavity.

In the first case, $L=5$, there are no superradiance (superfluorescence) pulses, and after several oscillations (Figure 4 a) the lasing becomes steady-state and consists of two independent monochromatic pairs of counterpropagating waves with different 
frequencies and equal intensities, $I|a|^{2}=5 \cdot 10^{-5}$. (For simplicity's sake, we do not take care of the quantum and thermal noises.) Due to the saturation effects (see, e.g., $[2,8])$, these fields burn two holes in the population inversion profile of a spectral line, which have a width $\Delta_{s} \approx 0.04$ (Figure 4 b). The spectral distribution of polarization of an active medium have two corresponding peaks with two times broader width (Figure 4C), as it should be for an oscillating dipole with a saturation nonlinearity placed into an external resonant monochromatic field.

In the second case, $L=6$, the lasing proceed above a so-called superradiant threshold, namely, the laser generates a sequence of phased-in pairs of oscillating mode superradiant pulses (Figure $5 a$ ). This sequence is almost periodic, and an average period $(\approx 230)$ is equal to a time interval it takes the pumping to restore an inversion level required for superradiance (Figure $5 a, b$ ). After each pulse the inversion becomes negative for a while in the two mode spectral channels due to generation of strong coherent fields in these channels (Figure $5 \mathrm{c}$ ), in accord with the energy conservation law. As is clearly seen from the dynamical spectrum of polarization (which is related to the dynamical spectrum of inversion via the Bloch-vector conservation law), the simultaneous superradiant pulses of the two neighboring lasing modes are coherently coupled by means of the spectral tails of each pulse (Figure $5 \mathrm{~d}$ ), although a spectral width of each pulse $(\sim 0.2)$ is approximately four times less than the distance between modes. Also, the dynamical spectrum of polarization makes it possible to resolve fine spectral structure of each mode superradiant pulse, because this spectrum is calculated straightforward and does not require a special computer Fourier analysis needed for evaluating the field dynamical spectrum. Note, that in this case (and in the next two cases of sections 5,6 as well) there is a short-term spontaneous symmetry breaking of the spatial profiles of field and polarization similar to that described in the previous sections.

\section{Active Medium with an Inhomogeneous Broadening of Spectral Line in a Combined DFB-FP Cavity with Asymmetric Modes}

To give a typical example of superradiant lasing in the case of asymmetric spectrum of modes, let us take real values, i.e., with zero phases, of both (equal) mirror reflection factors in a combined DFB-FP cavity considered in the previous section. Then, according to Figure 6 , the lasing regime is again quasiperiodic (with an average period $\approx 200$ ), but 
now it is formed by one wide-band superradiant mode and four narrow-band quasistationary modes modulated by the first one.

Note that to make the amplitudes of these four quasi-stationary lasing modes higher, we choose longer the cavity length as compared with the previous case shown in Figure 5 , namely, $L=7$ instead of $L=6$. The dynamical spectrum of polarization (Figure $6 c$ ) follows well the fine structure of modes and visualizes their interference. In particular, one can resolve an intermode coupling happen at the times when the superradiant pulses are emitted as well as the Rabi splitting of the most strong superradiant mode, which is also seen on the dynamical spectra of a population inversion (Figure 6d) and an inversion grating (Figure 6e).
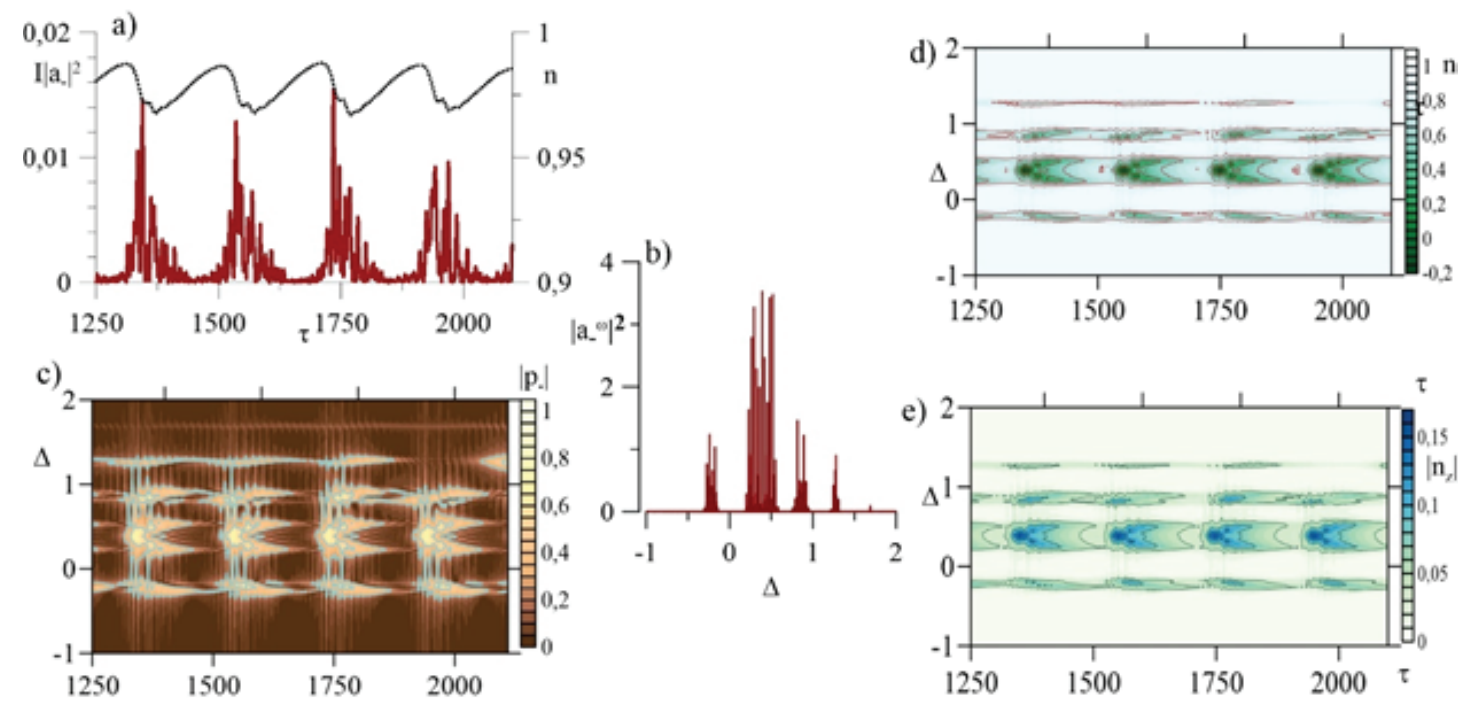

Figure 6: Superradiant lasing of a sample of length $L=7$ in a combined Fabry-Perot cavity with the reflection factor of both mirrors, $R=0.1$, and with the DFB coefficient $\beta=0.1\left(T_{E}=4.4\right)$ in the case of CW pumping $\left(n_{p}=1\right)$ and an inhomogeneously broadened spectral line: $\Gamma_{2}=2 \Gamma_{1}=0.02, \Delta_{0}=4$. (a) Oscillograms of the normalized output radiation intensities, $I\left|a_{ \pm}\right|^{2}$, and an oscillogram of the average inversion, $n$, (dotted curve). (b) Spectrum of the output fields at the left end of the laser. Dynamical spectrum (c) of the polarization, $\left|p_{-}(\Delta, \tau)\right|$, as well as (d) of the inversion, $|n(\Delta, \tau)|$, and (e) of the inversion grating, $\left|n_{z}(\Delta, \tau)\right|$, at the left facet of an active sample.

It can be shown that a valuable inversion grating (with the amplitude $\left|n_{z}\right| \sim 0.1$ ) in the present, previous and next cases influences essentially the asymmetric profiles of field and polarization in a cavity and increases the temporal variability of pulses making their difference more pronounced. The reason is that the phase and spatial position of the inversion grating differ from pulse to pulse with respect to a given DFB grating because the inversion grating has a lifetime $\left(T_{1}=100\right)$ less than the inter-pulse interval of time and is formed by the counterpropagating waves which are different in each subsequent superradiant pulse. 


\section{Active Medium with a Strong Inhomogeneous Broadening of Spectral Line in a Combined DFB-FP Cavity with Asymmetric Modes}

In the case of a strong inhomogeneous broadening, when a DFB-FP cavity provides a number of quasi-equidistant quasi-stationary lasing modes, there is a spectacular effect of the partial self-locking of these modes in the presence of one or more pulsed superradiant modes [5-7]. This effect takes place in a broad range of laser parameters in the absence of both the external modulation of its properties and any absorbers commonly employed for achieving a mode-locked regime of lasing a quasi-regular train of radiation pulses with durations shorter than the relaxation times of the population of energy levels and polarization of the active medium (cf., e.g. [8, 9]). The effect exists due to the superradiant pulses of modes with the highest growth rates which follow quasi-periodically with the period close to the pumping time, $T_{1}$, and burn out deep dips in the population inversion of the active medium, up to removing the population inversion in it, in certain spectral intervals and in certain time intervals. As a result, modes with the smaller growth rates and frequencies lying farther from the photonic bandgap are capable of a partial locking maintained by a coherent interaction with superradiant modes. In this case, if the reflection coefficients $R$ from the ends are not too small compared to the DFB parameter $\beta L$, another pulsed quasi-periodic component appears in emission with the time period approximately equal to the travel time of light in the cavity due to mode-locking of some modes; see an example in Figure 7, where the oscillogram (Figure $7 a$ ) of the field of twelve phased-in modes (Figure $7 \mathrm{~b}$ ) is shown.

The regular sequence of frequent self-mode-locking pulses and two sequences of rare pulses produced by two superradiant modes (two left modes in Figure $7 \mathrm{~b}$ ) are clearly seen in the dynamical spectrum of the polarization (Figure $7 \mathrm{c}$ ) and hardly resolved in the dynamical spectrum of the field (Figure 7d). Six intermediate lasing modes are not well phased in. It is interesting that many non-superradiant quasistationary modes would not appear in steady lasing in the absence of superradiant modes, because, according to the linearized Maxwell-Bloch equations, they would not have the growth rate. This operation regime is promising for generating a quasiregular train of high-power ultrashort coherent emission pulses upon CW pumping in the absence of additional elements or devices providing mode-locking. Complex features of the nonlinear mode interaction and inter-mode coherence may be revealed 

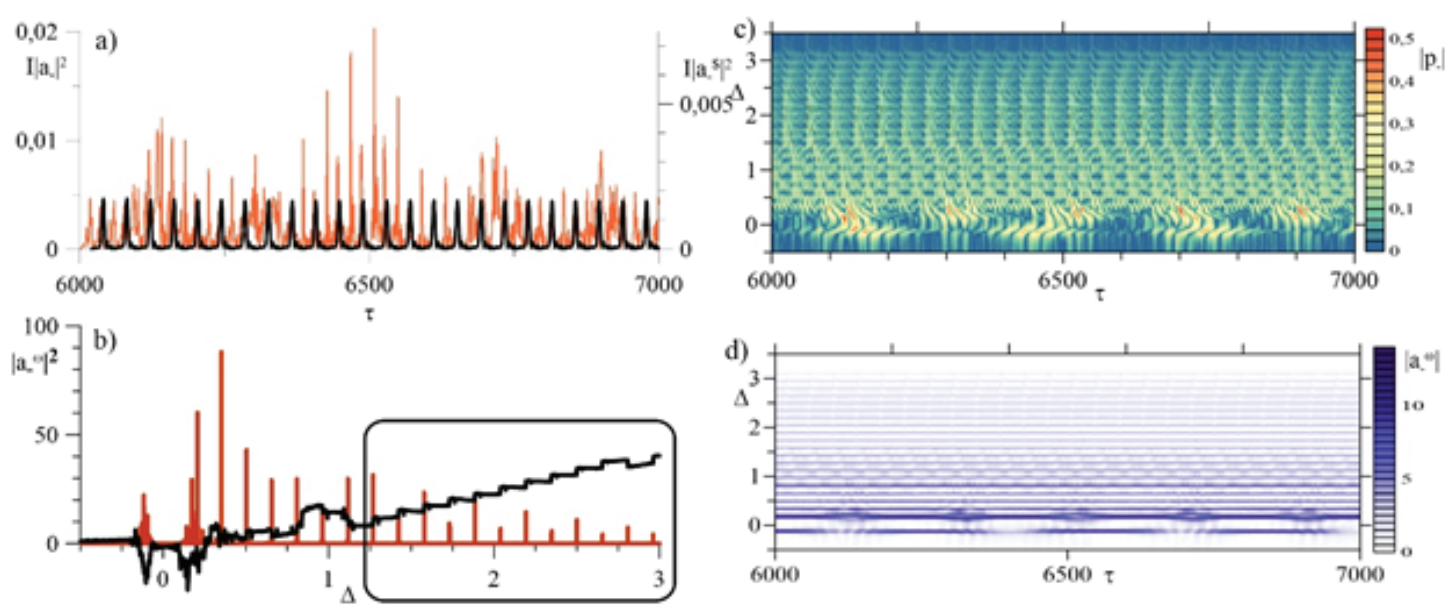

Figure 7: Superradiant lasing of a sample of length $L=20$ in a combined Fabry-Perot cavity with the real reflection factor of both mirrors, $R=0.15$, and with the DFB coefficient $\beta=\sqrt{3} / L\left(T_{E}=20\right)$ in the case of CW pumping $\left(n_{p}=0.9\right)$ and an inhomogeneously broadened spectral line: $\Gamma_{2}=2 \Gamma_{1}=0.02, \Delta_{0}=13$. (a) Oscillograms of the normalized output radiation intensities, $I\left|a_{ \pm}\right|^{2}$, (orange curve) and an oscillogramm of the field of twelve phased-in modes, $I\left|a_{-}^{s}\right|^{2}$, (dark curve). (b) Spectrum of the amplitude and phase (dark curve) of the output fields at the left end of the laser. Dynamical spectrum (c) of the polarization, $\left|p_{-}(\Delta, \tau)\right|$, and $(\mathrm{d})$ of the field, $\left|a_{-}^{\omega}(\Delta, \tau)\right|$, at the left facet of an active sample.

by means of the technique based on the spatial-temporal and/or spectral-temporal complex empirical orthogonal functions [6] evaluated from the polarization dynamics.

\section{Summary}

The examples considered demonstrate that the spatial-temporal dynamics of CW superradiant lasing in the dense ensembles of active centers with slow relaxation rate of dipole oscillations is extremely rich and may result in complicated, though quite regular spectral-temporal patterns of emission, with many potential applications in the dynamical spectroscopy and information processing. The analysis of the dynamical spectra of polarization of an active medium along with the dynamical spectra of electromagnetic field and population inversion allows one to describe in detail various regimes of the low-Q superradiant lasers, to find optimal conditions for the pulsed operation, and to give qualitative explanations of the major features of lasing processes. All these benefits are very important for managing the time profile, the correlation features, and the spectral properties of the generated radiation.

\section{Funding}

The work was partially supported by the Russian Foundation for Basic Research [a grant \# 16-02-00714], the Program of Fundamental Studies of the DPS RAS IV.2.6, 
'Fundamental Basis and Experimental Realization of Promising Semiconductor Lasers for Industry and Technologies', the Program of Fundamental Studies of the RAS Presidium P-1, 'Nanostructures: Physics, Chemistry, Biology, Basic of Technology', and the Competitiveness Program of National Research Nuclear University 'MEPhl'.

\section{Acknowledgements}

Author VI. V. Kocharovsky would like to thank MEPhl for the nice hospitality during his visit to the university.

\section{References}

[1] Kocharovsky, VI. V., Zheleznyakov, V. V., Kocharovskaya, E. R., et al. (2017). Superradiance: The principles of generation and implementation in lasers. PhysicsUspekhi, vol. 60, no. 4, pp. 345-384.

[2] Khanin, Ya. I. (2006). Fundamentals of Laser Dynamics. (Cambridge: Cambridge International Science Publishing).

[3] Cong, K, Zhang, Qi, Wang, Y., Timothy, G. N., Belyanin, A., Kono, J. (2016) Dicke superradiance in solids. JOSA B, Vol. 33, pp. C80-C101

[4] Kocharovskaya, E. R., Ginzburg, N. S., Sergeev, A. S., et al. (2016). Regimes of generation in low-Q distributed-feedback lasers with strong inhomogeneous broadening of the active medium. Radiophysics and Quantum Electronics, vol. 59, no. 6, pp. 484-500.

[5] Kocharovsky, VI. V., Belyanin, A. A., Kocharovskaya, E. R., et al. (2015). Superradiant lasing and collective dynamics of active centers with polarization lifetime exceeding photon lifetime. Advanced Lasers: Laser Physics and Technology for Applied and Fundamental Science, Springer Series in Optical Sciences, vol. 193, pp. 49-69.

[6] Kocharovskaya, E. R., Gavrilov, A. S., Kocharovsky, V. V., et al. (2016). Empirical mode with a variable spatial-temporal structure and the dynamics of superradiant lasers. Journal of Physics: Conference Series, vol. 740, pp. 012007-1-13.

[7] Kocharovsky, VI. V., Kalinin, P. A., Kocharovskaya, E. R., et al. (2013). Dynamics of the class $\mathrm{D}$ lasers based on the Bose-Einstein condensates, the submonolayer quantum dots and other exotic active media. Nonlinear Woves 2012 [in Russian], IAP RAS, Nizhny Novgorod, pp. 398-428.

[8] Ohtsubo, J (2017) Semiconductor Lasers: Stability, Instability and Choos (Series: Springer Series in Optical Sciences. V. 111). 
[9] Arkhipov, R.M., Arkhipov, M.V., Babushkin, I. V. (2015) On coherent mode-locking in a two-section laser. JETP Lett. vol. 101, pp. 149-153. 